\title{
Evaluation of Incompletely Staged Ovarian Malignancy: A Study in Western India
}

\author{
${ }^{1}$ Anusha Kamath, ${ }^{2}$ Pariseema Dave, ${ }^{3}$ Meeta Mankad
}

\begin{abstract}
Introduction: Incomplete initial surgery complicates subsequent management of ovarian tumors. This study aimed to study demographic and clinical factors associated with incompletely staged ovarian tumor patients.
\end{abstract}

Materials and methods: Twenty five patients who had undergone incomplete staging surgery for ovarian tumors outside Gujarat Cancer \& Research Institute, Ahmedabad, Gujarat, India, were included in this study. Their demographic and clinical features were studied in detail. Patients were either subjected to restaging or were referred for chemotherapy initially.

Results: Mean age of patients was 42 years (23-60 years); $64 \%$ of patients had abdominal pain as initial complaint; $64 \%$ patients were operated initially using a transverse incision. Only four patients were managed laparoscopically initially, others by laparotomy. Single/part of one ovary was removed in $60 \%$ of patients as part of initial surgery. Infundibulopelvic ligament was tied distally in $40 \%$ of patients. Omentectomy (Om) and lymphadenectomy were not done in most of the patients. Nineteen patients were found to have malignancy on final histopathological examination; 3 borderline malignancy; 1 granulosa cell tumor; and 2 benign tumors; $52 \%(n=13)$ patients were subjected to chemotherapy; $4 \%(n=1)$ were observed, and rest underwent restaging procedures.

Conclusion: Incompletely staged ovarian tumor patients, especially those with malignant histopathology, should be managed by gynecologic oncologists. The management needs to be individualized and cannot be a blanket therapy.

Keywords: Oncology, Ovarian cancer, Staging.

How to cite this article: Kamath A, Dave P, Mankad M. Evaluation of Incompletely Staged Ovarian Malignancy: A Study in Western India. J South Asian Feder Menopause Soc 2017;5(2):133-137.

Source of support: Nil

Conflict of interest: None

Date of received: 10 July 2017

Date of acceptance: 04 October 2017

Date of publication: December 2017

\section{INTRODUCTION}

Ovarian cancer is emerging as one of the most common malignancies affecting women in India and is rapidly

\footnotetext{
${ }^{1}$ Assistant Professor, ${ }^{2,3}$ Professor

1-3Department of Gynecologic Oncology, The Gujarat Cancer \& Research Institute, Ahmedabad, Gujarat, India

Corresponding Author: Pariseema Dave, Professor, Department of Gynecologic Oncology, The Gujarat Cancer \& Research Institute, Ahmedabad, Gujarat, India, Phone: +919824021410 e-mail: drpariseema@gmail.com
}

becoming a leading cause of mortality and morbidity. In India, during the period 2004 to 2005, proportion of ovarian cancer varied from 1.7 to $8.7 \%$ of all female cancers in various urban and rural population-based registries operating under the network of the National Cancer Registry Program (NCRP) of Indian Council Medical Research. ${ }^{1,2}$ In most of the population-based cancer registries in India, ovarian cancer is the third leading site of cancer among women, trailing behind cervical and breast cancer. ${ }^{3}$

Epithelial ovarian cancer has the worst prognosis among all gynecological malignancies. The overall 5-year survival is approximately $45 \%$, primarily due to the late stage at diagnosis of the disease. ${ }^{4}$

It needs to be managed by a multidisciplinary approach of good clinical evaluation, radiological investigations, chemotherapy, and surgical debulking. The number of comprehensive cancer centers in India that can offer such services is not enough, in comparison to the burden of the disease. Most of the patients initially present to general gynecologists or general surgeons who may not be adequately trained in oncology. Many patients receive suboptimal management due to logistical and socioeconomic constraints. A large number of patients belong to rural areas and have poor access to specialized healthcare. The cost of antineoplastic drugs is a major deterrent for many of the patients to continue with the treatment. Advanced stage of disease at diagnosis, inappropriate management, and poor compliance to therapy altogether are responsible for the dismal survival rates. Hence, through this study, we have attempted to analyze the factors contributing to the inappropriate initial management of the patients referred to a regional oncology center in Western India.

\section{MATERIALS AND METHODS}

This is a prospective study conducted at a regional cancer center from October 2015 to September 2016. All cases with ovarian malignancy who underwent some form of surgical intervention elsewhere were included in the study. A total of 32 patients were referred to our institute after initial incomplete surgery at other centers. None of the patients had received anticancer treatment during this period. Eight patients did not come for follow-up after first consultation and were hence excluded from the study. A total of 25 patients were included in the 
study. Approval from the Institutional Review Board was obtained. This was an observational study and hence no written consent was required. Most of the patients who had primary surgery outside had incomplete operative notes. An attempt was made to contact the surgeon and obtain further information wherever possible. All pathological slides of specimens obtained during the initial operation were reviewed at our institution to confirm the diagnosis of malignancy. All serum and radiological investigations and clinical findings were reviewed. A serum CA-125 level of $35 \mathrm{IU} / \mathrm{L}$ was taken as the cut-off value (Table 1).

The patients were clinically assessed and after requisite investigations, a decision for further management was made according to the National Comprehensive Cancer Network (NCCN) guidelines. After due consultation with patient and relatives, the patient was either planned for surgery or referred for chemotherapy. The NCCN guidelines define incomplete surgery by the following standards: (1) Intact uterus; (2) intact uterine adnexa; (3) retained omentum; (4) incomplete documentation of staging; or (5) residual disease. ${ }^{5}$ The patients were referred for chemotherapy in case of unresectable residual disease or in case of stages IA or IB with grade III differentiation according to NCCN guidelines. In a few cases, the patient was unwilling for a second surgery. The surgical procedures for restaging included peritoneal washing, careful exploration of the abdomen and pelvis, salpingo-oophorectomy, high position of ligation of the infundibulopelvic ligament, total abdominal hysterectomy (TAH), Om, peritoneal biopsy, and retroperitoneal lymph node dissection (PLND). Senior gynecologic oncologists performed all restaging operations. Frozen section was used when required. Carboplatin with paclitaxel was used for epithelial ovarian cancer and Bleomycin, Etoposide, and Cisplatin regimen was administered for granulosa cell tumor. All the cases that were referred for initial chemotherapy were reevaluated for debulking surgery after three cycles.

\section{RESULTS}

The mean age of the patients with ovarian cancer was 43.9 years (range $23-55$ years). Most of the women were in the perimenopausal age group. Only five women were postmenopausal. This is an important factor as the likelihood of malignancy increases in a postmenopausal woman with adnexal mass.

The most common presenting symptom was abdominal pain $(72 \%)$ followed by infertility $(12 \%)$ and menstrual abnormalities. All primary surgeries were planned except in one case where she was operated as an emergency with the suspicion of torsion. Majority $(72 \%)$ underwent
Table 1: Patient characteristics $(n=25)$

\begin{tabular}{|c|c|}
\hline Characteristics & Number (percentage) \\
\hline \multicolumn{2}{|l|}{ Abdominal incision } \\
\hline Transverse & $13(52)$ \\
\hline Infraumbilical midline vertical & $4(16)$ \\
\hline Supraumbilical midline vertical & $1(4)$ \\
\hline Laparoscopy & $7(28)$ \\
\hline \multicolumn{2}{|l|}{ Operating surgeon } \\
\hline Gynecologist_-private clinic & $15(60)$ \\
\hline Gynecologist-teaching hospital & $5(20)$ \\
\hline Surgeon & $3(12)$ \\
\hline Oncosurgeon & $2(8)$ \\
\hline \multicolumn{2}{|l|}{ Serum CA-125 level } \\
\hline Not done & $14(56)$ \\
\hline Normal & $7(28)$ \\
\hline Elevated (35-500 IU/I) & $4(16)$ \\
\hline \multicolumn{2}{|l|}{ Histology } \\
\hline Serous & $16(64)$ \\
\hline Mucinous & $2(8)$ \\
\hline Borderline serous & $2(8)$ \\
\hline Borderline mucinous & $2(8)$ \\
\hline Endometrioid & $1(4)$ \\
\hline Granulosa cell tumor & $2(8)$ \\
\hline \multicolumn{2}{|l|}{ Assessment of omentum } \\
\hline Not assessed & $19(76)$ \\
\hline Gross assessment & $1(4)$ \\
\hline Omental sampling & $4(16)$ \\
\hline Infracolic Om & $1(4)$ \\
\hline \multicolumn{2}{|l|}{ Management at our institute } \\
\hline Chemotherapy & $16(64)$ \\
\hline Restaging & $7(28)$ \\
\hline Observation & $2(8)$ \\
\hline
\end{tabular}

CA-125 = Carbohydrate antigen 125

laparotomy and the others were operated laparoscopically. Table 1 summarizes the patient characteristics.

Of the 18 women who underwent laparotomy, nearly half were operated by a transverse skin incision. The type of incision is important as a midline vertical incision up till the xiphoid is considered as appropriate for adequate exposure, proper exploration of the abdominal cavity, and complete staging.

Primarily, a general gynecologist has operated majority of the patients; $20 \%$ were operated at a teaching hospital and $12 \%$ were operated by a general surgeon. Three patients were referred from an in vitro fertilization (IVF) center and were incidentally discovered to have ovarian malignancy during the workup for infertility.

Peritoneal washings were taken only in five cases, which were negative for malignancy. Peritoneal biopsy was not taken in any case. Lymph nodes were only assessed in one case and lymphadenectomy was not done in any of the cases. There was intraoperative tumor spillage in two cases. The tumor was aspirated before removal in two cases.

Tumor markers were not done in majority of the patients. It was elevated in four patients, though not to 


\begin{tabular}{|c|c|c|c|c|}
\hline Malignancy & $\begin{array}{l}\text { Primary } \\
\text { surgery }\end{array}$ & $\begin{array}{l}\text { Provisional } \\
\text { stage }\end{array}$ & Restaging & $\begin{array}{l}\text { Final } \\
\text { stage }\end{array}$ \\
\hline $\begin{array}{l}\text { Granulosa } \\
\text { cell tumor }\end{array}$ & $\begin{array}{l}\mathrm{LSO}+\mathrm{Rt} \\
\text { ovarian biopsy }\end{array}$ & la & $\begin{array}{l}\text { B/I PLND and } \\
\text { PAND }\end{array}$ & la \\
\hline $\begin{array}{l}\text { Granulosa } \\
\text { cell tumor }\end{array}$ & Cystectomy & la & $\begin{array}{l}\text { TAH + BSO + } \\
\text { Om + B/l PLND }\end{array}$ & la \\
\hline $\begin{array}{l}\text { Borderline } \\
\text { serous }\end{array}$ & LSO & la & $\begin{array}{l}\text { TAH + RSO + } \\
\text { Om + PLND }\end{array}$ & la \\
\hline $\begin{array}{l}\text { Serous } \\
\text { papillary }\end{array}$ & Cystectomy & la & $\begin{array}{l}\text { TAH + BSO + } \\
\mathrm{Om}+\mathrm{B} / \mathrm{PLND}\end{array}$ & $\mathrm{Ilb}$ \\
\hline $\begin{array}{l}\text { Borderline } \\
\text { serous }\end{array}$ & RSO & la & $\mathrm{B} / \mathrm{PLND}+\mathrm{Om}$ & la \\
\hline Serous & USO & la & $\begin{array}{l}\text { TAH + RSO + } \\
\text { Om + B/I PLND }\end{array}$ & la \\
\hline
\end{tabular}

TAH: Total abdominal hysterectomy; LSO: left salpingooophorectomy; RSO: Right salpingo-oophorectomy; BSO: Bilateral salpingo-oophorectomy; B/l: bilateral; PLND: Pelvic lymph node dissection; PAND: Para-aortic node dissection; Om: Omentectomy

a value more than 500 . This may have been attributed to nonmalignant conditions like endometriosis or pelvic inflammatory disease. The omentum was not assessed in two-thirds of the patients.

Epithelial ovarian variety was the most common histology. Almost 70\% were of serous epithelial type followed by mucinous cyst-adenocarcinoma and borderline variety. One case each of endometrioid epithelial tumor and granulosa cell tumor was identified. Frozen section was performed in four patients.

Most of the patients were referred for chemotherapy either due to inoperable disease, higher grade, or patient's unwillingness to undergo a second surgery. Six patients were taken up for restaging (Details have been provided in Table 2). Both the patients who were put on observation were of the borderline variety. Only one patient [resented to us with residual disease after primary incomplete surgery. She was referred for chemotherapy as the disease was at the vault and clinically unresectable.

One of the patients was upstaged. Two patients were nulliparous and referred from an IVF center. Hence, fertility sparing surgery was done.

Delay in referral was encountered in five patients. It was mostly on the part of the patient due to financial issues, indecision, or temporary symptomatic relief by initial surgery. No deaths or major complications were reported during the course of the study.

\section{DISCUSSION}

Surgery is important to determine the correct disease stage and to remove as much tumor as possible in ovarian cancer patients. Several studies have shown that ovarian cancer patients who underwent surgery by a gynecologist had better survival compared with patients who underwent surgery by general surgeons. ${ }^{6-10}$ Subsequently, it was suggested that surgery by a gynecologic oncologist would improve survival further., ${ }^{9,11,12}$ This has been found to be associated especially with International Federation of Gynecology and Obstetrics stage III disease. ${ }^{11,13}$

The pivotal study by Young et $\mathrm{al}^{14}$ demonstrated the deficiency in comprehensive staging of presumed early ovarian cancer. Since then, sparse studies confirmed this initial observation showing a considerable lack regarding adherence to staging guidelines, especially outside of specialized gynecologic oncology centers. ${ }^{9,10}$

Advanced stage of disease at diagnosis, inappropriate management, and poor compliance to therapy all together are responsible for the dismal survival rates. There is a need to objectively assess these factors in the context of ovarian cancer management in Indian scenario. ${ }^{6}$

The standard of care for ovarian cancer is thorough surgical staging with optimal cytoreduction followed by a platinum-based chemotherapy, if there is a significant risk of recurrence. A midline vertical incision up to the xiphoid process is mandatory to gain adequate access to the upper abdomen. Peritoneal fluid or peritoneal wash should be sent for cytology and the entire abdomen should be explored to look for the extent of disease including metastases. Debulking of all visible tumors (including TAH and bilateral salpingo-oophorectomy), Om, pelvic, and para-aortic node dissection, and representative biopsies from different parts of the abdominal cavity should be done. A thorough staging procedure can upstage up to $30 \%$ of the clinically early stage ovarian cancers and save them from inadequate treatment. ${ }^{14}$

We observed that all patients operated outside the cancer institute were not staged appropriately. In many developed countries, all known or suspected ovarian cancer patients are referred to trained gynecological oncologists for surgery..$^{10}$ Also in some countries there is a concept of guest operations as referral of each and every case is not possible. ${ }^{15}$ There is ample evidence that patients with advanced ovarian malignancies have significant survival advantage when a gynecologic oncologist is involved in their care. ${ }^{10-13}$ In India, trained gynecological oncologists are handful in number. The general gynecologists need to be trained and oriented about oncology. We observed that some patients were initially managed at gynecologic teaching institutes, where adherence to oncologic guidelines was not adhered. This is in contradiction to expected management at least at a teaching center. Also two patients were initially managed by trained oncologist (one each a surgical and gynecologic oncologist). Patient managed by surgical oncologist had grade III malignancy and thus had required adjuvant chemotherapy. Second, patient managed by gynecologic oncologist underwent diagnostic laparoscopy, where inoperable disease was found. Both these patients were subjected to chemotherapy. 
In our study, we found that a small subset of patients were initially managed laparoscopically. Laparoscopic approach is not yet established as standard of care, as far as managing ovarian cancer patients are concerned, although these patients were presumed benign cases. Also most of the laparotomies were done by a transverse abdominal incisions, which limits complete abdominal cavity evaluation. A midline vertical incision is far more convenient whenever staging procedure is to be undertaken.

The commonest "missing steps" in initial surgery of most of these patients were adequate peritoneal assessment, omental assessment, and lymph node assessment. These are crucial steps in evaluation of ovarian cancer patients as they can upstage a presumed early stage malignancy.

The commonest final histopathology in our subset of patients was serous malignancy, which is in consonance with the general distribution of ovarian malignancy. There were four borderline tumors on review of initial histopathology. Both the patients with serous borderline tumor underwent restaging, which is recommended to avoid relapse. ${ }^{16}$ One of the patients wanted to undergo IVF and was referred to us for staging after being incidentally discovered as a case of borderline malignancy. The other lady had completed her childbearing and was willing for completion of surgery. Both of them remained stage Ia after restaging. Borderline mucinous cases were kept under observation after explaining the risk of recurrence. Some studies confirm that overall survival remains unaffected and relapse mainly occurs in the remaining ovary and can be easily managed by surgery. ${ }^{16}$

All perimenopausal women having ovarian/adnexal mass need to be thoroughly assessed and requisite "malignancy index" scoring may be applied, if need be.

Several diagnostic tools are available nowadays, like risk of malignancy index, risk of ovarian malignancy algorithm, Assessment of Different NEoplasias in the adneXa model, ${ }^{17-19}$ to predict likelihood of malignancy in adnexal masses. These tools should be utilized by general gynecologist to avoid missing diagnosis of ovarian malignancies. This would obviate incomplete surgeries and would benefit patients overall. All postmenopausal patients should preferably be not managed by gynecologist lacking frozen section facilities. All preoperative investigations may fail to pick a diagnosis of malignancy except on frozen section, which too has its own limitations.

\section{CONCLUSION}

We recommend that ovarian malignancy being the most lethal of all gynecological malignancies needs to be better addressed. All teaching institutes should adhere to basic oncologic principles, especially that of staging, and should attempt to keep gynecologic oncologist available for a possible comprehensive staging procedure.

Laparoscopy is not yet established as standard approach to address ovarian malignancy patients and should be avoided whenever the index of suspicion is high. Midline vertical incision should be used while undertaking all staging procedures, since it allows for a thorough inspection of entire abdominal cavity and all requisite staging procedures like multiple peritoneal biopsies, Om, and para-aortic lymph node sampling in certain cases. Frozen section facilities should be preferably available at least at all teaching postgraduate institutes to avoid late diagnosis of ovarian malignancy patients. Oncologic training should be essential part of postgraduate teaching so as to orient all general gynecologists toward oncology. And most important, general surgeons and gynecologists should be adequately trained in referral and importance of staging.

\section{REFERENCES}

1. National Cancer Registry Programme. Two year report of population based cancer registries, 2004-2005. Incidence and distribution of cancer. Bengaluru: Indian Council of Medical Research (ICMR); 2008.

2. Murthy NS, Shalini S, Suman G, Pruthvish S, Mathew A. Changing trends in incidence of ovarian cancer - the Indian scenario. Asian Pac J Cancer Prev 2009 Jan;10(6):1025-1030.

3. National Cancer Registry Program. Consolidated report of population based cancer registries 2001-2004. Bengaluru: Indian Council of Medical Research; 2006.

4. Jemal A, Siegel R, Ward E, Murray T, Xu J, Smigal C, Thun MJ. Cancer statistics, 2006. CA Cancer J Clin 2006 Mar-Apr;56(2): 106-130.

5. National Comprehensive Cancer Network (NCCN). Clinical practice guidelines in oncology. Ovarian Cancer. Fort Washington (PA): NCCN; 2014. p. 2.

6. Basu P, De P, Mandal S, Ray K, Biswas J. Study of 'patterns of care' of ovarian cancer patients in a specialized cancer institute in Kolkata, eastern India. Indian J Cancer 2009 JanMar;46(1):28-33.

7. Timmers PJ, Zwinderman AH, Coens C, Vergote I, Trimbos JB. Understanding the problem of inadequately staging early ovarian cancer. Eur J Cancer 2010 Mar;46(5):880-884.

8. Junor E. The impact of specialist training for surgery in ovarian cancer. Int J Gynecol Cancer 2000 Jan-Feb;10(Suppl 1):16-18.

9. Orr JW Jr. What constitutes the "optimal" treatment environment of women with gynecologic cancer? Gynecol Oncol 2003 Apr;89(1):1-3.

10. Engelen MJA, Kos HE, Willemse PH, Aalders JG, de Vries EG, Schaapveld M, Otter R, van der Zee AG. Surgery by consultant gynecologic oncologists improves survival in patients with ovarian carcinoma. Cancer 2006 Feb;106(3):589-598.

11. Grabowski JP, Harter P, Buhrmann C, Lorenz D, Hils R, Kommoss S, Traut A, du Bois A. Re-operation outcome in patients referred to a gynecologic oncology center with presumed ovarian cancer FIGO I-IIIA after sub-standard initial surgery. Surg Oncol 2012 Mar;21(1):31-35. 
12. Earle CC, Schrag D, Neville BA, Yabroff KR, Topor M, Fahey A, Trimble EL, Bodurka DC, Bristow RE, Carney M, et al. Effect of surgeon specialty on processes of care and outcomes for ovarian cancer patients. J Natl Cancer Inst 2006 Feb;98(3):172-180.

13. Elit LM, Bondy SJ, Paszat LP, Holowaty EJ, Thomas GM, Stukel TA, Levine MN. Surgical outcomes in women with ovarian cancer. Can J Surg 2008 Oct;51(5):346-354.

14. Young RC, Decker DG, Wharton JT, Piver MS, Sindelar WF, Edwards BK, Smith JP. Staging laparotomy in early ovarian cancer. JAMA 1983 Dec;250(22):3072-3076.

15. Peters IT, van Haaften C, Trimbos JB. If the mountain does not come to Mohammad: the significance of guest operations for early stage ovarian cancer. J Gynecol Surg 2014 Oct;30(5):265-272.

16. Romeo M, Pons F, Barretina P, Radua J. Incomplete staging surgery as a major predictor of relapse of borderline ovarian tumor. World J Surg Oncol 2013 Jan;11:13.
17. Simsek HS, Tokmak A, Ozgu E, Doganay M, Danisman N, Erkaya S, Gungor T. Role of a risk of malignancy index in clinical approaches to adnexal masses. Asian Pac J Cancer Prev 2014 Oct;15(18):7793-7797.

18. Karlsen MA, Sandhu N, Høgdall C, Christensen IJ, Nedergaard L, Lundvall L, Engelholm SA, Pedersen AT, Hartwell D, Lydolph M, et al. Evaluation of HE4, CA125, risk of ovarian malignancy algorithm (ROMA) and risk of malignancy index (RMI) as diagnostic tools of epithelial ovarian cancer in patients with a pelvic mass. Gynec Oncol 2012 Nov;127(2): 379-383.

19. Sayasneh A, Ferrara L, Cock BD, Saso S, Al-Memar M, JohnsonS, Kaijser J, Carvalho J, Husicka R, Smith A, et al. Evaluating the risk of ovarian cancer before surgery using the ADNEX model: a multicentre external validation study. Br J Cancer 2016 Aug;115(5):542-548. 